\title{
La question des revues dans la science ouverte : une approche fonctionnelle
}

\author{
Auteurs \\ Joachim Schöpfel \\ Université de Lille SHS \\ Laboratoire GERiiCO \\ BP 60149 \\ 59563 Villeneuve d'Ascq Cedex \\ joachim.schopfel@univ-lille.fr
}

06.88.35.01.47

Biographie

Joachim Schöpfel est MCF en SIC à l'Université de Lille, membre du laboratoire GERiiCO et consultant partenaire de la société Ourouk, Paris. II est auteur de plus de 150 publications et d'études dans le domaine de l'information scientifique, en particulier sur l'édition scientifique, l'open access, les données de recherche. Il est membre du Comité pour la Science Ouverte (collège données).

Photographie

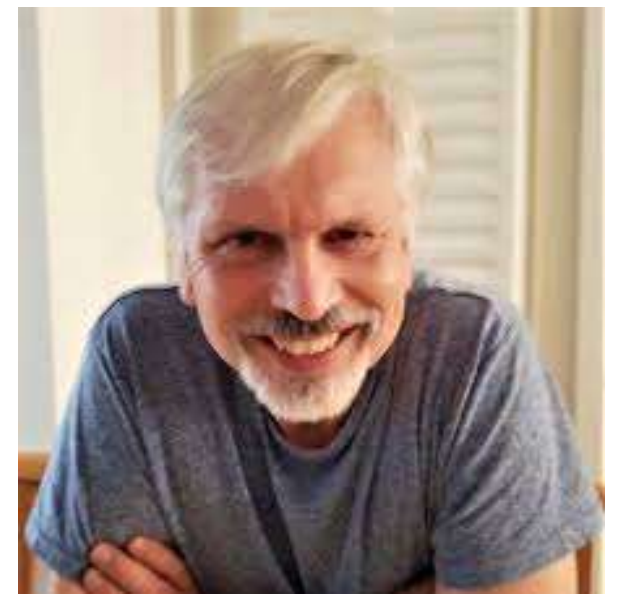

\section{Renaud Fabre}

Université Paris 8 Vincennes - Saint-Denis

Laboratoire Paragraphe

2 rue de la Liberté

93526 Saint-Denis

renaud.fabre01@gmail.com

Biographie 
Renaud Fabre est professeur émérite des Universités en Sciences économiques à l'Université de Paris VIII (Vincennes - Saint-Denis). Il en a été le président de 1996 à 2001. Diplômé de la section "Service public " de l'Institut d'études politiques de Paris, Renaud Fabre est également docteur d'État en Sciences économiques. II a été directeur de la Direction de I'Information scientifique et technique du CNRS de 2013 à 2017.

Photographie

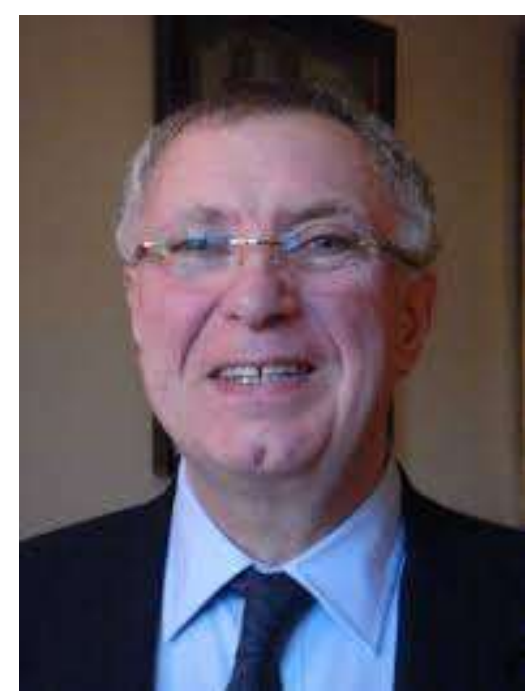

\section{Résumé}

La science ouverte est devenue une priorité de premier rang de la politique de recherche de l'Etat français. Parmi les axes de cette politique, se trouve l'ouverture des données de recherche et des publications scientifiques. A partir de ses fonctions traditionnelles (qualité, propriété intellectuelle, diffusion, conservation), l'article interroge l'impact de la politique de la science ouverte sur les revues scientifiques, en particulier dans trois domaines: leur rôle dans le fonctionnement des dispositifs d'évaluation et de suivi (fonction politique), leur rôle dans le fonctionnement des dispositifs de données, en particulier pour l'acquisition et l'exploitation des données massives par l'industrie de l'information (fonction "big data"), et leur rôle dans le fonctionnement économique des éditeurs, agrégateurs, agences etc. (fonction économique). Dans la mesure où l'accès aux revues devient gratuit, la question se pose si l'usage des revues ne devient pas le véritable produit des plateformes, par le biais des informations générées.

\section{Mots-clés}

Accès libre, édition scientifique, revues scientifiques, science ouverte

\section{English title}

The issue of journals in open science: A functional approach

\section{Abstract}

Open science has become a top priority of the French government's research policy. One of the main areas of this policy is the opening up of research data and scientific publications. Based on its traditional functions (certification, registration, dissemination, archive), the article questions the impact of open science policy on scientific journals, in particular in three areas: their role in the functioning of evaluation and monitoring systems (political function), their role in the functioning of 
data systems, in particular for the acquisition and exploitation of massive data by the information industry (big data function), and their role in the economic functioning of publishers, aggregators, agencies etc. (economic function). Since access to journals becomes free, the question arises as to whether the use of journals does not become the real product of the platforms, through the information generated.

\section{Keywords}

academic publishing, open access, open science, scientific journals

\section{Introduction}

La science ouverte est devenue une priorité de premier rang de la politique de recherche de l'Etat français, qui décline ainsi sa propre version des principes européens et internationaux en cours de généralisation. Parmi les axes de cette politique, se trouve l'ouverture des données de recherche et des publications scientifiques. Pour développer le libre accès aux publications, deux modèles s'affrontent depuis quelques années en Europe et offrent une première image des "systèmes " éditoriaux qui structurent la science ouverte :

- Le modèle de Liège, porté par l'ancien recteur de l'Université de Liège, Bernard Rentier, et adopté en mai 2018 par le Parlement de la Wallonie-Bruxelles ${ }^{1}$ : le dépôt obligatoire des articles scientifiques subventionnés par des fonds publics dans une archive institutionnelle (Rentier 2019).

- L'initiative OA2020 portée par la Max-Planck-Gesellschaft: la conversion du modèle traditionnel de l'abonnement vers le modèle " auteur-payeur » dans des revues en libre accès (Schimmer et al. 2015).

Stevan Harnad, l'un des pionniers du libre accès, avait décrit deux options : la voie verte (green road) pour le dépôt dans une archive ouverte, et la voie dorée (gold road) pour la publication dans une revue en libre accès (Harnad et al. 2004, cf. aussi Suber 2012). Le modèle de Liège relève de la voie verte, tandis que l'initiative OA2020 est une variante de la voie dorée. Malgré toutes leurs différences, les deux modèles ont un point en commun : loin de régler tous les problèmes (cf. Ericksson 2018) et loin d'être des modèles disruptifs, ils s'appuient sur le marché des revues scientifiques dont les imperfections et asymétries sont connues (Dewatripont et al. 2006). Ces systèmes font penser davantage à une économie d'exploitation de marques qu'à une économie de la prestation, avec des dysfonctionnements et dérives par rapport à la tarification (Vajou et al. 2009).

Chacun à leur façon, ces systèmes sont encore mal décrits et, de plus, il n'y a pas de modèle pour la transition. L'harmonisation à l'échelle internationale ou européenne risque d'être compliquée, car les positions respectives des Etats sont souvent peu lisibles et comparées : chaque pays n'a pas les mêmes priorités ni les mêmes ressources financières pour la science ouverte, ce qui risque de conduire à " une géopolitique de la science ouverte assez complexe » (Chartron 2018a).

Faute d'un modèle unique ou encore d'un système descriptif uniformément reconnu pour décrire toutes les pratiques, chaque État développe beaucoup d'initiatives et dispense des conseils pour faciliter la transition des revues scientifiques associées à son propre écosystème éditorial, pour aller au plus vite vers le libre accès. Les mesures en tous genres foisonnent tout en relevant d'un nombre croissant de leviers d'action, et forment un très large éventail : un récent rapport commandé par OpenAIRE (Johnson et al. 2017) fait état de 26 recommandations différentes, dont la plupart sont soit

\footnotetext{
1 Texte du décret http://www.pfwb.be/le-travail-du-parlement/doc-et-pub/documents-parlementaires-et-
} decrets/documents/001597235 
d'ordre économique (création de fonds institutionnels pour payer les $A P C^{2}$, réorganisation des budgets de bibliothèques, transparence des tarifs et coûts de publication, efficience des mécanismes de paiement etc.) soit politique (soutien des archives ouvertes, renforcement des consortia, monitoring) soit juridique (création d'un environnement légal favorable au libre accès, standardisation des licences, contractualisation de la transformation vers le libre accès etc.). Force est donc de constater que ces politiques nationales parallèles sont chacune vouées à réaliser "la science ouverte dans un seul pays " comme on disait autrefois du socialisme en URSS : leur stratégies et objectifs sont différents, certaines politiques apparaissent complémentaires, d'autres incompatibles.

Analysées simultanément et sous le même prisme, ces actions nationales parallèles peu connectées, laissent craindre un télescopage ou, au mieux, risquent d'entretenir la confusion : en effet, au-delà du principe général selon lequel la politique de la science ouverte poursuit l'objectif de rendre librement accessibles " les publications scientifiques » au sens large, les politiques de science ouverte privilégient avant tout, dans des proportions et modalités diverses, des démarches nationales de soutien à des éventails spécifiques de revues scientifiques, comme vecteur d'amélioration de la communication scientifique (de Silva \& Vance 2017). Définies comme publications périodiques qui rendent compte des résultats de la recherche sous forme d'articles rédigés par les chercheurs et publiés à la suite d'un examen par les pairs en bonne et due forme (Johnson et al. 2018) ${ }^{3}$, les revues sont censées remplir quatre fonctions, et ceci depuis leur création il y a plus de 350 ans ("I'ombre d'Oldenburg ", cf. Guédon 2001, aussi Mabe 2008 et Rentier 2019) :

- le contrôle de qualité des travaux des scientifiques,

- leur diffusion,

- leur archivage à plus long terme,

- et l'attribution aux auteurs de la propriété intellectuelle de leurs contenus.

Dans un environnement en pleine mutation, on commence à évaluer les risques de cette transition. Sans disposer d'une représentation systémique de ces risques (Piwowar et al. 2018), et sans disposer d'outils standards globaux de définition des outils de décompte des publications en open access (Science-Metrix 2018), on voit se multiplier les analyses énumérant les causes de risques : l'inflation des frais de publication ${ }^{4}$, une concentration toujours plus forte dans le secteur de l'édition scientifique et de l'industrie de l'information (Posada \& Chen 2018), une nouvelle fracture numérique entre pays riches et pauvres (Sotudeh \& Ghasempour 2018) ou encore l'uberisation de la science et le développement d'un "platform capitalism » autour de quelques grands acteurs (Chan 2019); en d'autres termes, " we observe nominally philanthropic foundations collaborating with for-profit firms to build the One Platform to Rule Them All » (Mirowski 2018)5.

Nul doute qu'à partir de là, la perte de cohérence globale du processus nous éloigne de la vision humaniste de la Déclaration de Berlin de 2003 du libre accès comme " une source universelle de la connaissance humaine et du patrimoine culturel ayant recueilli l'approbation de la communauté scientifique ".

Dans le fonctionnement de l'écosystème émergent de la science ouverte, le rôle des revues scientifiques a entamé une mutation, et d'autres enjeux se font sentir et contribuent à une

\footnotetext{
${ }^{2} \mathrm{APC}=$ article processing charges, frais de publication facturés par des éditeurs pour diffuser un article en libre accès (modèle " auteur-payeur »)

3 "periodicals carrying accounts of research written by the investigators themselves and published after due peer review"

${ }^{4}$ Le Wellcome Trust a constaté début 2018 une inflation de 7-11\% entre 2016 et 2017

https://wellcome.ac.uk/funding/managing-grant/wellcome-and-coaf-open-access-spend-2016-17

5 Traduction : « nous observons des fondations nominalement philanthropiques qui collaborent avec des entreprises à but lucratif pour construire la Plate-forme unique pour les gouverner toutes »
} 
transformation en profondeur du paysage de l'édition scientifique, allant jusqu'à une remise en question radicale de l'intérêt intrinsèque des revues : "Personne ne veut que les revues meurent. Mais peut-être qu'on devrait... " ${ }^{6}$. Peut-être la " quadrature éditoriale " (Chartron 2018b) autour des valeurs et des fonctions fondamentales de l'édition scientifique (libre accès, indépendance, qualité, pérennité) est en train de devenir une mission impossible ?

Nous allons décrire ici trois fonctions qui aujourd'hui prennent de l'importance, à partir des fonctions traditionnelles et parfois, à leur détriment, pour faire émerger de nouveaux systèmes éditoriaux :

- La fonction politique ;

- La fonction « big data » ;

- La fonction économique.

Il s'agit d'une première approche d'une analyse de l'impact de la science ouverte sur les revues scientifiques sous l'aspect fonctionnel, d'un état des lieux et de quelques observations d'un processus en cours dont l'issue est aujourd'hui des plus incertaines.

\section{La fonction politique}

Un enjeu majeur des revues scientifiques est leur fonction politique, c'est-à-dire leur rôle dans le fonctionnement des dispositifs d'évaluation et de suivi (monitoring). A titre d'illustration, quelques exemples. L'Observatoire des Sciences et Techniques ${ }^{7}$ produit entre autres des indicateurs scientométriques afin de contribuer à la connaissance des activités de recherche en France et dans le monde, à l'évaluation des activités de recherche et à l'évaluation des politiques en faveur de la recherche et de l'innovation. La part mondiale des publications et les indices d'impact ou de spécialisation scientifique figurent parmi les principaux indicateurs de l'OST ; ils sont calculés à partir de données liées aux publications d'articles dans des revues scientifiques, en particulier les affiliations, les domaines et les citations.

Selon les dernières études de l'OST sur l'évolution de la production scientifique dans le monde et en France (OST 2018 et 2019), la France est le $7^{\mathrm{e}}$ pays produisant le plus de publications scientifiques en 2015 mais est passée du $5^{\mathrm{e}}$ au $7^{\mathrm{e}}$ rang depuis 2000, derrière l'Inde et le Japon. Dans la même période, d'autres pays européens ont enregistré des évolutions quantitatives ou qualitatives plus importantes. Les indicateurs du laboratoire espagnol Scimago ${ }^{8}$, à partir des chiffres de Scopus, confirment le constat de l'OST (figure 1). Entre 1998 et 2018, le nombre annuel des publications françaises a presque doublé, passant de 55098 à $106278^{9}$. En parallèle, la part de la production française passe de 4,76\% à 3,89\% de la production globale. Il s'agit d'un processus dans la continuité.

\footnotetext{
${ }^{6}$ Egon Willighagen (2019). Plan S: Less publications, but more quality, more reusable? Yes, please. https://chem-blaics.blogspot.com/2019/02/plan-s-less-publications-but-more.html

7 OST https://www.hceres.fr/fr/observatoire-des-sciences-et-techniques-ost

8 https://www.scimagojr.com/

${ }^{9}$ Documents citables (essentiellement des articles scientifiques)
} 


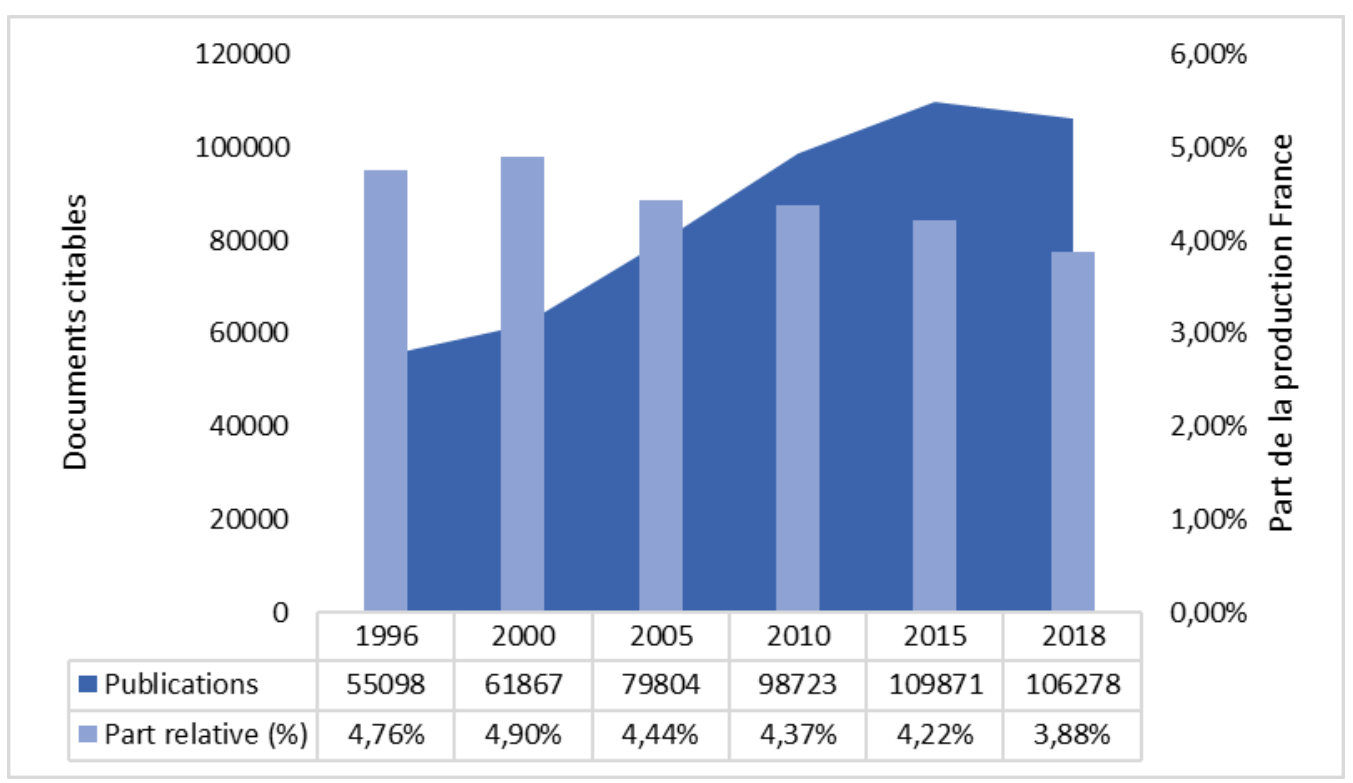

Figure 1. Publications françaises 1996-2018 (source : Scimago/Scopus)

Quant aux revues publiées en France et indexées dans Scopus, leur nombre augmente légèrement entre 2010 et 201, de 500 à 531 (+6\%), mais le nombre d'articles baisse, de 31684 à $27066(-15 \%)$. Par rapport à l'ensemble des revues dans Scopus, l'édition française passe de 2,36\% à 2,15\% en termes de revues et de $1,71 \%$ à $1,08 \%$ en termes d'articles.

L'analyse longitudinale de l'évolution du nombre de revues par pays entre 1950 et 2013 montre que le taux de croissance des revues en français $(0,54 \%)$ est largement en-dessous de la moyenne globale $(2,91 \%)$; ce taux positif à long terme cache une baisse réelle à partir de 1970 (Wang et al. 2017). L'écart entre les revues en anglais et les autres s'élargit. II s'agit d'une érosion lente et continue dont les corollaires sont une marginalisation croissante dans les études scientométriques et rankings internationaux. L'étude de Wang et al. établit un lien entre le lancement de nouveaux titres, le développement économique et une politique favorable à l'édition scientifique.

Les revues scientifiques jouent un rôle similaire et significatif dans l'évaluation des établissements scientifiques. Parmi les six indicateurs du Shanghai Ranking ${ }^{10} \mathrm{~d}^{\prime}$ universités, trois sont directement liées aux revues scientifiques : le nombre de chercheurs fortement cités, le nombre d'articles publiés dans les revues Nature et Science, et le nombre d'articles indexés par le Web of Science (source Clarivate Analytics). Le poids de ces trois critères dans le résultat du classement est de $60 \%$.

L'impact politique de ce ranking est réel, aussi bien pour la stratégie et la communication institutionnelle ${ }^{11}$ que pour la politique nationale de recherche, comme l'a constaté Enserink (2007)pour la loi sur l'autonomie des universités ${ }^{12}$; lors de la publication du classement 2019 , le Ministère salue " la position de la France en Gème place mondiale (...) (le) classement de Shanghai (...) constitue un indicateur important du rayonnement scientifique des universités françaises ${ }^{13}$; le

\footnotetext{
${ }^{10}$ ARWU http://www.shanghairanking.com/index.html

11 Cf. I'article sur le classement de l'Université de Lille dans La Voix du Nord du 15 août 2019

https://www.lavoixdunord.fr/625109/article/2019-08-15/classement-de-shanghai-2019-lille-entre-dans-les-400-premieres$\underline{\text { universites }}$

12 Citation: "France's poor showing in the Shanghai rankings-it had only two universities in the first top 100-helped trigger a national debate about higher education that resulted in a new law, passed last month, giving universities more freedom".

${ }^{13}$ Communiqué 15 août 2019 http://www.enseignementsup-recherche.gouv.fr/cid144243/publication-du-classement-deshanghai-2019-reaction-de-frederique-vidal.html
} 
Ministère souligne comme enjeu la visibilité de "la position scientifique de la France dans la compétition internationale " et la mise en évidence du "potentiel scientifique des établissements d'enseignement supérieur ", et rappelle l'importance des affiliations institutionnelles.

Le poids des revues dans l'autre grand classement international des universités est moins important mais toujours sensible. Dans la méthodologie d'évaluation de 1200 universités par Times Higher Education ${ }^{14}$, les revues scientifiques comptent pour $36 \%$ du résultat, $6 \%$ pour le nombre d'articles et $30 \%$ pour le nombre de citations. La source de données ici est Scopus d'Elsevier.

A ce jour, le développement et la mise en œuvre de la politique de la science ouverte n'a pas fondamentalement modifié ce rôle politique des revues, au contraire. Le volet de l'ouverture de la publication scientifique (open access) concerne essentiellement les revues scientifiques.

Toutefois, conformément aux observations initiales, la dissémination de "l'effet open access » est inégale ; l'interprétation de ce vaste mouvement d'inégale appropriation de l'OA, est à peine entrepris (Robinson-Garcia et al. 2019). Les deux initiatives les plus marquantes au niveau international, $\mathrm{OA} 2020^{15}$ et le Plan $\mathrm{S}^{16}$, visent la transformation du marché des revues. Le rôle central que jouent dans ce contexte les identifiants comme le DOI (pour les articles) et ORCID (pour les auteurs) laisse penser que l'évaluation à partir de la publication d'articles dans des revues scientifiques ne sera sans doute pas mise en question en profondeur par la science ouverte, sauf à devenir plus transparente et ouverte, au sens réutilisable.

Quant aux "altmetrics ", il s'agit d'indicateurs alternatifs aux différents facteurs d'impact mais souvent, directement ou indirectement, liés aux revues scientifiques (Williams 2016). Aussi, une grande partie des indicateurs de suivi pour évaluer les effets de cette politique (monitoring) est calculée à partir des articles de revues ou d'autres informations issues de l'édition de revues (Schöpfel \& Prost 2019). Reste à établir l'incidence de leur développement sur les tendances corrélatives à la publication ouverte.

\section{La fonction « big data »}

Un deuxième enjeu des revues scientifiques est leur rôle dans le fonctionnement des dispositifs de données, en particulier pour l'acquisition et l'exploitation des données massives par l'industrie de l'information. Là où l'analyse traditionnelle met en avant le rôle des revues pour la qualité et la diffusion des résultats de la recherche, l'environnement des données massives (big data) transforme les revues en un dispositif complexe de génération d'information à fort potentiel d'exploitation et de valorisation. Tout devient données, comme par exemple :

- La composition et l'activité du comité de rédaction ;

- La soumission de manuscrits ;

- Le processus et les acteurs d'évaluation ;

- Les affiliations des auteurs ;

- Les références citées et liens vers d'autres sites ;

- Les illustrations (images, tableaux, graphiques...) ;

- Les abonnements ;

\footnotetext{
14 THE World University Rankings https://www.timeshighereducation.com/world-university-rankings/world-universityrankings-2019-methodology

${ }^{15}$ Max Planck Digital Library https://oa2020.org/

16 cOAlition-S, un consortium d'agences, de fonds de recherche et de fondations privées https://www.coalition-s.org/
} 
- Les publicités, les annonces de conférences et de formation ;

- Les citations et altmetrics des articles publiés (statistiques d'usage, réseaux sociaux, etc.) ;

- Les liens vers des entrepôts de données ;

- Les liens vers des systèmes d'information recherche.

Cette liste est loin d'être exhaustive, et on pourrait continuer par la terminologie scientifique, le multilinguisme, les informations institutionnelles, sans même parler du contenu scientifique des articles.

A partir des exemples de Wiley et d'Elsevier, Posada et Chen (2018) ont décrit en détail comment cette stratégie d'intégration verticale risque de créer de nouvelles dépendances et d'impacter des décisions individuelles aussi bien que des stratégies institutionnelles. Herb (2019) analyse le même développement comme l'émergence d'une variante du " data capitalism » basée sur la mise en place d'un système d'exploitation de services fortement intégrés, qui couvrent l'ensemble du processus scientifique, d'un service de recommandations de revues pour publier un article jusqu'aux services de veille scientifique, de conseil stratégique et de monitoring, avec le risque d'un effet de "singlehoming ", qui enfermerait le chercheur dans l'utilisation exclusive d'un seul service (cf. Pillot et Marty 2019).

Dans ce système d'exploitation, les revues jouent un rôle central dans la mesure où une grande partie des données massives à disposition de l'industrie de l'information scientifique et technique est liée directement ou indirectement aux revues. Certes, les revues ne sont pas la source unique pour l'information sur la recherche, mais l'illustration des services d'Elsevier laisse penser qu'elles en sont toujours la source principale (figure 2).

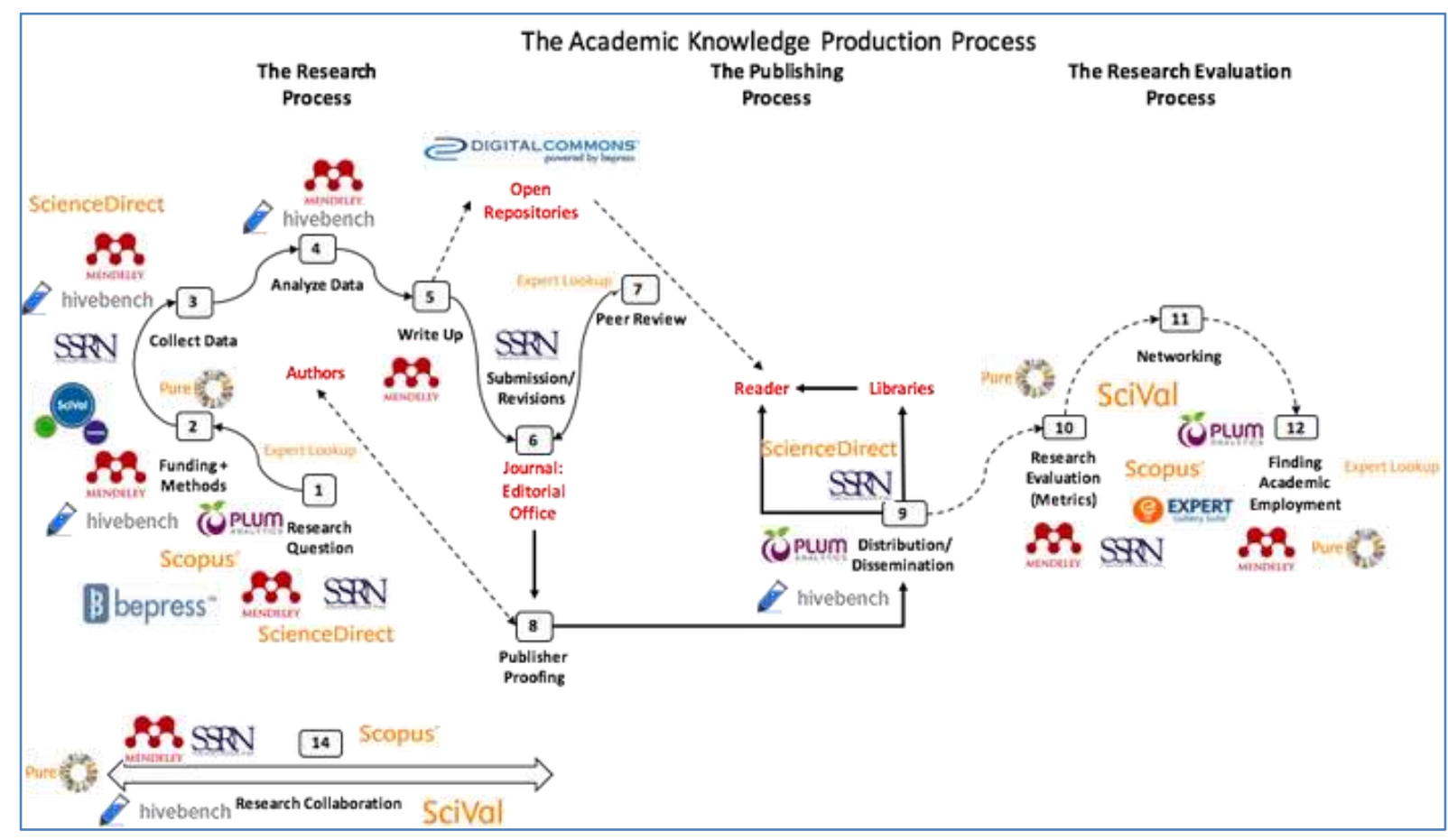

Figure 2. L'intégration des services d'Elsevier (source : Posada et Chen 2018)

Sans mettre en question cette fonction, le développement de la science ouverte contribue probablement à renforcer l'effet du big data sur les revues, pour deux raisons au moins. D'une part, 
l'application des principes FAIR (Wilkinson et al. 2016) à l'édition des revues facilitera sans doute, par le biais de la normalisation des métadonnées et identifiants, l'exploitation automatique des données de revues. D'autre part, la diffusion massive et sans restriction d'articles génère des données massives d'utilisation, sur les plateformes d'édition et d'agrégation, sur les plateformes institutionnelles, dans des réseaux sociaux etc. Le cas des articles de données (data papers) donne un aperçu sur le potentiel des nouvelles technologies, associées aux nouvelles infrastructures de la science ouverte - un univers de données et de métadonnées, fortement normalisé, avec une production semi-automatique, destinée (aussi) à une exploitation automatique; mais au centre se trouvent toujours les revues scientifiques (Schöpfel et al. 2019).

C'est sans doute un facteur systémique majeur de renouvellement des modèles, en faveur de la publication ouverte : le développement de normes et standards numériques globaux est une condition du développement de ces modèles.

\section{La fonction économique}

La fonction économique des revues scientifiques, c'est-à-dire, leur rôle dans le fonctionnement économique des éditeurs, agrégateurs, agences etc., est sans doute la fonction la mieux connue, la plus controversée aussi. La valeur du marché STM est estimée à 25,7 milliards \$ en 2017 dont 38,7\% pour les revues, avec une croissance annuelle de 3 à $4 \%$ (McShea 2018). Selon le dernier rapport annuel STM, il y aurait 42500 revues scientifiques vivantes avec peer review, dont 33100 titres en anglais (avec 3 millions d'articles par an) et 9400 titres dans d'autres langues (22\%), avec un taux de croissance de 5 à $6 \%$ (Johnson et al. 2018).

La production de revues scientifiques est liée à l'évolution des secteurs publics et privés de la recherche et développement, dont la croissance en 2019 est estimée à $+3,9 \%$, pour un volume total de 2,3 billions $\$$ dans les pays les plus riches ${ }^{17}$. Il paraît inutile de rappeler la nature dysfonctionnelle du marché des revues, la crise des revues, les mouvements de boycott des bibliothécaires et chercheurs (The Cost of Knowledge ${ }^{18}$ ), le piratage massif $\left(\right.$ Sci-Hub ${ }^{19}$ ) ou militant (affaire Aaron Swartz ${ }^{20}$ ) et l'impact de " l'oligopole » des grands éditeurs (cf. Dewatripont et al. 2006, Larivière et al. 2015). Frazier (2001) a analysé les négociations des big deals sous forme de la théorie des jeux et a comparé les relations entre bibliothèques et éditeurs au dilemme du prisonnier: "Il ne s'agit pas d'une situation "gagnantgagnant". Une fois que notre fidélité a été consolidée au point où le Big Deal devient indispensable, le résultat est un gagnant-perdant "; côté perdant, il s'agit des bibliothèques et de leurs établissements mais aussi des agrégateurs et éditeurs proposant un catalogue plus restreint. Ainsi, Frédéric Bouchard, président du conseil d'administration du consortium interuniversitaire Érudit, a énumérés parmi les défis de la plateforme la « diminution des budgets d'acquisition des bibliothèques (et la) concentration des capitaux entre les mains d'une poignée de grands éditeurs commerciaux ${ }^{21}$.

L'essentiel ici est d'insister sur le fait que plus d'un tiers de la valeur du marché STM - environ 10 milliards \$ - est générée par les revues, avec une croissance relativement stable et au-dessus des taux d'inflation des pays développés. A ce jour, l'impact de la science ouverte paraît incertain et parfois contradictoire. Schimmer et al. (2015) estime qu'une transformation disruptive du modèle des abonnements vers un modèle open access avec APC peut se faire sans risque (" there is enough money already circulating in the global market »); ce calcul s'avère pour l'instant optimiste et inexact. Le

\footnotetext{
${ }^{17}$ https://www.rdmag.com/news/2019/03/global-funding-forecast-predicts-growth-r-d-spending-worldwide

18 http://thecostofknowledge.com/

19 https://fr.wikipedia.org/wiki/Sci-Hub

${ }^{20}$ https://fr.wikipedia.org/wiki/Aaron Swartz

21 Érudit : Rapport annuel 2016-2017 https://www.erudit.org/rapport/2017/
} 
débat autour du Plan $\mathrm{S}$ de la cOAlition $\mathrm{S}^{22}$ a démontré à quel degré les grands éditeurs ont compris les nouvelles conditions de la science ouverte comme autant d'opportunités pour consolider et développer leur chiffre d'affaires, tandis que les petits éditeurs et sociétés savantes ont plus de mal à faire face (Wise \& Estelle 2019). Néanmoins, il ne faut probablement pas surestimer l'effet immédiat du Plan S sur l'édition scientifique car sa portée semble assez limitée : seulement peu de publications - 3-5\% de la production totale - seraient impactées à l'heure actuelle (Hampson 2019), et la mise en œuvre du plan paraît à ce jour retardée voire diluée.

Un certain nombre d'études ont tenté de déterminer le " juste prix " d'une revue ou d'un article, à partir d'une analyse comptable des dépenses fixes et variables. Récemment, Grossmann \& Brembs (2019) ont ainsi estimé que les revenus générés par la publication des revues étaient cinq fois supérieurs aux dépenses directement liées à leur production, du moins pour les grands éditeurs et les " big deals ", la différence correspondant aux frais de promotion et de diffusion, aux investissements, aux dividendes etc. La part des dépenses de production varie d'un éditeur à l'autre; aussi, pour certains éditeurs, la publication des revues constitue la source principale des revenus tandis que pour d'autres, elle n'est qu'une source de revenus parmi d'autres, moins ou peu importante, ou une activité déficitaire.

La science ouverte n'a pas modifié cette fonction économique des revues scientifiques en profondeur. Les revues restent à ce jour une source significative de revenus dans l'écosystème de l'information scientifique. L'évolution des politiques scientifiques de la Commission européenne, des Etats et des organismes et établissements, ainsi que l'évolution des critères des agences de financement obligent les éditeurs à adapter leurs offres de services et leurs modes de fonctionnement et de production, pour réduire des coûts de production et de diffusion, mais aussi pour créer de nouvelles sources de chiffre d'affaires, afin de développer le libre accès aux résultats de la recherche. De nouveaux modèles économiques émergent, dont certains à caractère provisoire et temporaire, pour une période de transition vers les « $100 \%$ open access ».

Mais malgré leur grande diversité la plupart de ces modèles et recommandations ont un point commun : le fait que les revenus soient directement conditionnés par la production et la diffusion des revues. Rares sont les modèles alternatifs qui affaibliraient ou feraient disparaître la fonction économique des revues ; citons par exemple le développement d'un dispositif de preprints en amont ou à la place des revues (Green 2019), ou évoquons l'option " close or combine " pour réduire des coûts par l'arrêt d'une revue ou la fusion de deux ou plusieurs revues (Wise \& Estelle 2019). Tous les autres modèles - APC, crowdfunding, syndication, read/publish, ainsi que les nouveaux services pour les auteurs (structuration, mise en forme, traduction... cf. Mehra 2019) etc. - s'appuient sur la production des revues et maintiennent, voire renforcent, leur fonction économique.

\section{Un écosystème dynamique et complexe}

Le plan national pour la science ouverte en France définit la science ouverte comme " la diffusion sans entrave des publications et des données de la recherche (qui) s'appuie sur l'opportunité que représente la mutation numérique (...) ». Dans le cadre de l'Union Européenne, la France s'engage " pour que les résultats de la recherche scientifique soient ouverts à tous, chercheurs, entreprises et citoyens, sans entrave, sans délai, sans payement ${ }^{23}$. Cet engagement mobilise tous les acteurs de l'enseignement supérieur et de la recherche, et leurs projets, initiatives et stratégies créent un écosystème dynamique et complexe, dont le rôle est non négligeable pour les choix stratégiques des éditeurs et l'évolution de

\footnotetext{
22 cOAlition S https://www.coalition-s.org/

${ }^{23} \mathrm{https}$ ://www.ouvrirlascience.fr/category/science ouverte/
} 
leurs modèles d'affaires (Gadd et al. 2018), mais dont l'impact à plus long terme est encore difficile à mesurer.

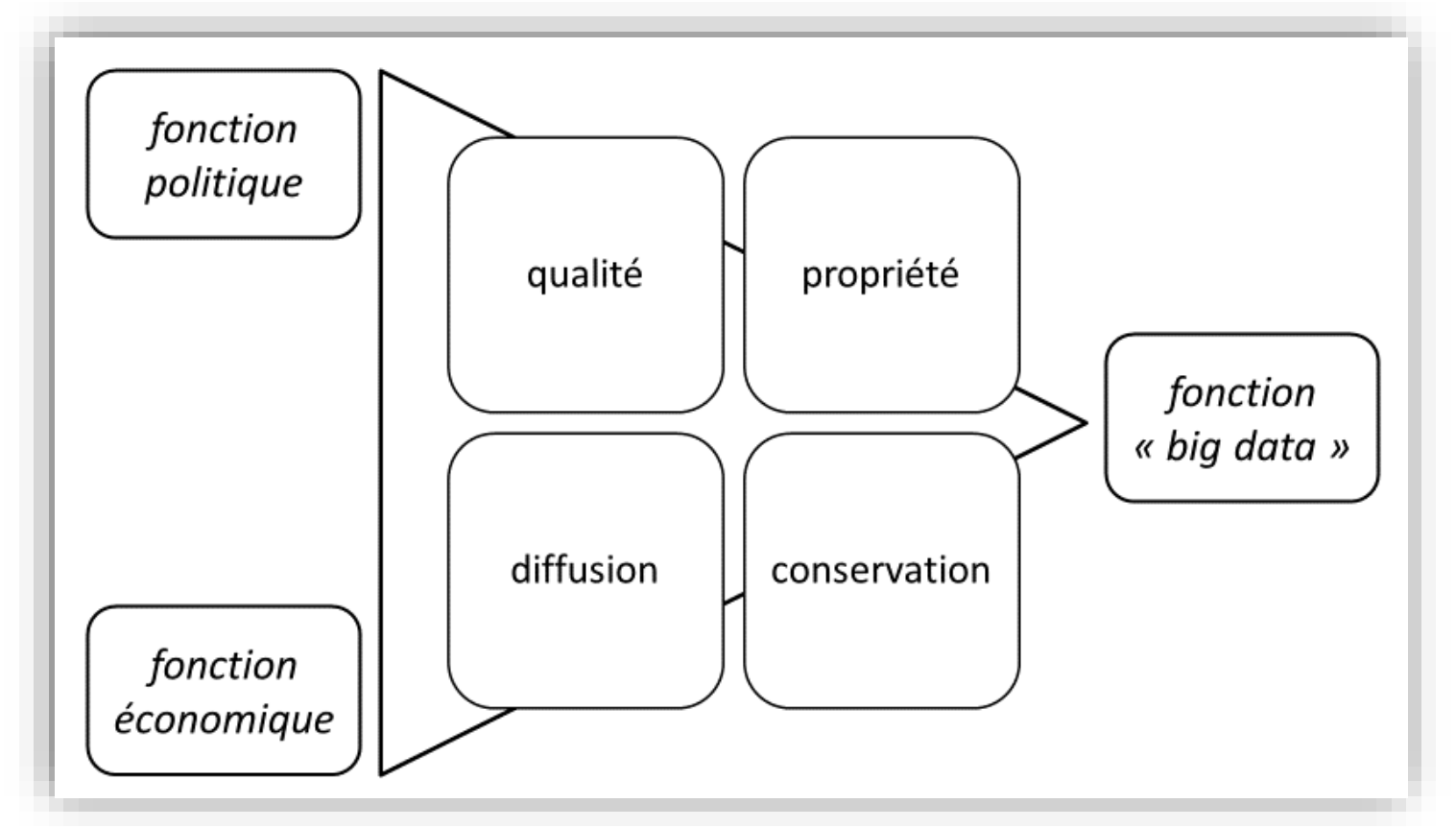

Figure 3. Les fonctions traditionnelles et nouvelles des revues scientifiques

Malgré les discours de rupture et de transformations " disruptives ", la politique de la science ouverte de l'Union européenne et de ses Etats membres, des principales agences de financement et des grands organismes de recherche ne semble pas remettre en question le modèle des revues scientifiques (figure 3). Elle conforte plutôt la fonction des revues pour la diffusion des résultats de la recherche et a tendance à renforcer leur fonction politique, par le biais de l'avantage des citations et des altmetrics (Piwowar et al. 2018, AlRyalat et al. 2019).

Les verrous pour l'ouverture des publications sont plutôt culturels ou systémiques, liés au manque d'incitatifs ; l'évaluation de la recherche est l'enjeu central qui détermine la stratégie des éditeurs, le comportement des chercheurs et le choix politique des institutions (Guédon et al. 2019). Cependant, même une politique en faveur des archives institutionnelles comme en France (cf. le droit d'exploitation secondaire de la Loi numérique et le soutien au dispositif HAL) ne remet pas en question le principe même de la revue. Ceci sera d'autant plus vrai à partir du moment où ces archives seront alimentées directement à partir des plateformes de revues des éditeurs ou via les systèmes $\mathrm{d}^{\prime}$ information recherche (cf. licence nationale Elsevier).

Le Plan S est généralement interprété comme défavorable aux éditeurs en SHS où les APC sont peu acceptés, aux sociétés savantes et aux petits éditeurs indépendants, avec moins de ressources et réserves pour se mettre en conformité avec les critères du plan $\mathrm{S}$ et faire face à une éventuelle baisse de revenus pendant la transition ${ }^{24}$. L'impact de la science ouverte, au moins à court terme, pourrait bien être une concentration et consolidation du marché des revues sans effet de rupture du modèle de la revue; " le plan S ne clarifie pas l'avenir des sociétés savantes qui vivent des abonnements à leurs éditions, et dont le prix est généralement très raisonnable " (Rentier 2019). Un commentaire sur le site

${ }^{24}$ A titre d'exemple, la prise de position de l'Open Access Scholarly Publishers Association https://oaspa.org/oaspafeedback-on-plan-s-implementation-guidance/ et le débat au sein de la Society for Scholarly Publishing https://scholarlykitchen.sspnet.org/ 
de la Society for Scholarly Publishing avertit que " la différence entre la transition actuellement plus lente vers le libre accès et la transition plus rapide du Plan $S$ pourrait faire la différence entre la survie et l'extinction ${ }^{25}$ - oui mais survie ou extinction d'une certaine catégorie d'éditeurs, pas du modèle des revues en général.

II ne faut pas perdre de vue que les stratégies et initiatives comme le Plan $\mathrm{S}$ sont une variable parmi d'autres dans le paysage de l'édition des revues scientifiques, avec un impact, certes, mais en convergence ou compétition avec $d$ 'autres facteurs, tels que les systèmes nationaux et internationaux d'évaluation et de classement institutionnel, les procédures de sélection d'articles, l'évolution du secteur de la recherche ou plus largement le contexte économique.

Ainsi, une étude pour le UK Arts and Humanities Research Council mentionne plusieurs menaces du modèle d'affaires des revues au Royaume Uni, comme, par exemple, le taux d'inflation, les restrictions du financement public des universités et organismes de recherche, et l'évolution du taux de change défavorable à la Livre Sterling (Fyfe et al. 2017). Tout cela n'a aucun rapport avec la science ouverte. En France, on pourrait citer d'autres variables, comme la stagnation de l'emploi dans la recherche publique ${ }^{26}$, les modalités d'attribution de subventions aux laboratoires (avec l'incitation de publier dans des revues internationales), une prévalence d'éditeurs et de structures éditoriales de petite taille, notamment en SHS, et un financement insuffisant des services d'IST publics (Ourouk 2018).

Comment la science ouverte va-t-elle modifier les quatre fonctions traditionnelles des revues scientifiques ? Elle renforcera probablement, via l'ouverture, leur fonction centrale pour la diffusion des résultats, au moins aussi longtemps qu'elle ne contribuera pas au développement massif d'une bibliodiversité avec d'autres vecteurs et canaux. En revanche, dans la mesure où une partie de la politique de la science ouverte explore des alternatives à l'évaluation organisée par les éditeurs de revues (peer review), elle affaiblira probablement leur fonction pour assurer un " contrôle qualité " des résultats diffusés. Même constat pour les deux autres fonctions. L'incitation à déposer les articles de revues dans des archives institutionnelles va progressivement diminuer l'importance des revues pour la conservation des résultats de la recherche, surtout quand les dispositifs (comme c'est le cas pour HAL) peuvent garantir une conservation numérique à long terme. Quant à leur rôle pour la protection de la propriété intellectuelle des auteurs, il faudra sans doute attendre un peu pour mesurer l'effet de la Loi numérique, non pas en termes de chiffres mais en termes juridiques.

Un problème plus général, pas spécifiquement français, est le piratage massif des contenus de plateformes de revues et leur diffusion via Sci-Hub ou d'autres serveurs qui pose également un problème d'intégrité et de sécurité des réseaux et sites scientifiques, notamment universitaires. Néanmoins, à ce jour, l'effet sur la publication et le nombre des revues, ou encore sur la politique de la science ouverte reste incertain, dans la mesure où ce piratage est justement alimenté par la production et la diffusion des revues.

Dans l'environnement de la science ouverte, une autre variable pourrait jouer un rôle dans le développement des revues : la critique, voire le refus des "big deals", y compris sous la forme de " contrats transformatifs ", et le développement de la bibliodiversité27. Il est trop tôt pour évaluer l'effet sur les revues de quelques " big deals » non conclus ou non reconduits, comme l'Université de Californie. Certes, il y aura (il y a déjà) un effet sur le marché de I'IST, sur les revenus des éditeurs et leurs modèles d'affaires. Mais tout cela changera-t-il ou remettra-t-il en question le modèle intrinsèque des revues scientifiques, leurs fonctions et leurs rôles dans l'écosystème de la science

\footnotetext{
${ }^{25}$ https://scholarlykitchen.sspnet.org/2019/02/11/with-thousand-of-pages-of-feedback-on-the-plans-s-implementationguidance-what-themes-emerged-that-might-guide-next-steps/

${ }^{26}$ Source : OCDE https://data.oecd.org/rd/government-researchers.htm

27 Cf. I'Appel de Jussieu https://jussieucall.org/
} 
ouverte ? L'augmentation du nombre des revues va-t-elle ralentir ? L'importance des revues pour l'évaluation des institutions et des chercheurs va-t-elle faiblir ? Trouvera-t-on des alternatives aux revues pour la diffusion des résultats de la recherche - pas seulement dans quelques niches mais massivement? En 2017, on pouvait lire sur Twitter que « dans moins de dix ans, Elsevier ne s'attend plus à gagner de l'argent avec les publications, mais avec la gestion des données et les services de recherche ${ }^{28}$. Quid alors des revues?

\section{Conclusion}

La science ouverte est « un concept polyvalent et susceptible d'être défini, selon les contextes et les acteurs impliqués, de multiples façons " (Vanholsbeeck 2017). Comme " idée programmatique " ou déclinée sous forme d'objectifs opérationnels, ce nouveau paradigme de la politique de recherche se retrouve en tension avec d'autres objectifs, tels que le positionnement dans les classements internationaux, la valorisation des innovations ou encore, pour la France, la défense de la francophonie et du rayonnement de la langue française.

Vecteur central de la communication scientifique, la revue scientifique est au cœur des stratégies et scénarios pour l'ouverture des publications des résultats de la recherche. L'analyse fonctionnelle montre que la politique de la science ouverte a un impact complexe et parfois contradictoire sur la publication des revues. Elle paraît affaiblir certaines fonctions (dont le contrôle qualité et la conservation), tout en renforçant d'autres (dont notamment la diffusion mais aussi les fonctions politique et données), sans pour autant, du moins à présent et d'une manière générale, menacer directement la fonction économique de la publication des revues.

Notre étude n'est qu'une première tentative d'évaluer l'impact de la politique en faveur de la science ouverte sur le modèle des revues scientifiques. Ce modèle existe depuis plus de 350 ans; il a montré que sa flexibilité, sa diversité et sa capacité d'adaptation, sont des gages de survie à toutes sortes de crises et de " ruptures ». Pour autant, ce n'est pas une garantie pour l'avenir.

Le débat récent autour du Plan $\mathrm{S}$ a montré que tous les acteurs- agences, institutions, bibliothèques, éditeurs etc. - étaient d'accord sur l'objectif et le principe du libre accès aux articles de revues. Les controverses portaient sur les délais et les conditions, pas sur le support principal de la diffusion des résultats, c'est-à-dire les revues. Aussi, le monitoring du développement du libre accès aux publications concerne essentiellement les revues, en faisant abstraction (au moins pour l'instant) d'autres vecteurs de la communication scientifique. C'est une bonne nouvelle pour le modèle des revues, pas nécessairement pour tous les acteurs (éditeurs, agrégateurs etc.).

Mais peut-être faudra-t-il un jour poser la question du « open access " d'une autre manière et faire le lien avec l'économie numérique. La politique de la science ouverte préconise la gratuité et l'accessibilité maximale des publications, ce qui se traduit notamment en une diversité de stratégies et de modèles pour ouvrir l'accès aux revues scientifiques. Or, par rapport aux services libres et gratuits sur le web, il y a une règle qui dit que " si c'est gratuit, c'est vous êtes le produit " (if it's free, you're the product). Pourquoi cette règle ne s'appliquerait-elle pas au libre accès des revues scientifiques ?

Nous avons évoqué le rôle des revues dans le fonctionnement des dispositifs de données, en particulier pour l'acquisition et l'exploitation des données massives par l'industrie de l'information, comme l'une des fonctions émergentes des revues scientifiques. Et si, à terme et favorisée par le développement du libre accès et de l'interopérabilités des dispositifs, cette fonction deviendrait la raison d'être principale des revues ? Où les revues (ouvertes, libres, gratis...) serviraient essentiellement à " voir » qui fait quoi avec qui et quand ? Où leur valeur principale serait la génération d'information sous forme

${ }^{28}$ Alexandre Grossmann @SciPubLab 11 mai 2017 
de données massives qui, exploitées et transformées, permettraient la production de nouveaux services d'intelligence économique et scientifique ? Dans ce cas, les revues auraient définitivement quitté l'ombre d'Oldenburg.

\section{Bibliographie}

ALRYALAT, S. A. et al., " The impact of the open-access status on journal indices: a review of medical journals ». F1000Research, 2019, n 8, p. 266. https://doi.org/10.12688/f1000research.17979.1

CHAN, L. Platform Capitalism and the Governance of Knowledge Infrastructure. In : Digital Initiative Symposium, University of San Diego, April 29-30, 2019. https://zenodo.org/record/2656601

CHARTRON, G., "L'Open science au prisme de la Commission européenne ». Education et sociétés, 2018, vol. 41, n¹, p. 177-193 (a). https://doi.org/10.3917/es.041.0177

CHARTRON G. Géopolitique de l'open access. In : ICOA 3rd International Colloquium on Open Access, 28-30 November 2018, Rabat, Maroc (b). https://hal.archives-ouvertes.fr/hal-01930281

DE SILVA, P., VANCE, C. Scientific Scholarly Communication: The Changing Landscape. Springer, 2017.

DEWATRIPONT, M., et al. Study on the economic and technical evolution of the scientific information markets in Europe. DG Research European Commission, 2006.

ENSERINK, M., " Who ranks the university rankers? " Science, 2007, vol. 317, n 5941, p. 1026-1028. https://doi.org/10.1126/science.317.5841.1026

ERIKSSON, J., "Offsetting: no big deal? " Nordic Perspectives on Open Science, 2018, n 1, p. 1-11. https://doi.org/10.7557/11.4430

FRAZIER, K., "The librarians' dilemma: Contemplating the costs of the 'big deal' ». D-Lib Magazine, 2001, vol. 7, n³. https://doi.org/10.1045/march2001-frazier

FYFE, A. et al. Untangling Academic Publishing: A History Of The Relationship Between Commercial Interests, Academic Prestige And The Circulation Of Research. University of St Andrews, 2017. https://doi.org/10.5281/zenodo.546100

GADD, E., FRY, J., CREASER, C., "The influence of journal publisher characteristics on open access policy trends ». Scientometrics, 2018, vol. 115, n³, p. 1371-1393. https://doi.org/10.1007/s11192-018$\underline{2716-8}$

GREEN, T., "Is open access affordable? Why current models do not work and why we need internetera transformation of scholarly communications ». Learned Publishing, 2019, vol. 32, n 1, p. 13-25. https://doi.org/10.1002/leap.1219

GROSSMANN, A., ET BREMBS, B. Assessing the size of the affordability problem in scholarly publishing. PeerJ Preprints, 2019. https://doi.org/10.7287/peeri.preprints.27809v1

GUEDON, J.-C. A l'ombre d'Oldenburg : Bibliothécaires, chercheurs scientifiques, maisons d'édition et le contrôle des publications scientifiques. In : ARL Meeting, Toronto, May 2001. https://hal.archivesouvertes.fr/halshs-00395366/

GUEDON, J.-C. et al. Future of Scholarly Publishing and Scholarly Communication. DG Research and Innovation European Commission, 2019. https://publications.europa.eu/en/publication-detail/Lpublication/464477b3-2559-11e9-8d04-01aa75ed71a1

HAMPSON, G. OSI Policy Perspective: Plan S \& the quest for global open access. Open Scholarship Initiative, 2019. http://osiglobal.org/2019/03/13/osi-policy-perspective-plan-s-the-quest-for-globalopen-access/ 
HARNAD, S., et al., "The Access/Impact Problem and the Green and Gold Roads to Open Access ". Serials Review, 2004, vol. 30, n 4, p. 310-314. https://doi.org/doi: 10.1016/j.serrev.2004.09.013

HERB, U. Steering science through Output Indicators \& Data Capitalism. In : 23rd Congress of the European Society of Veterinary and Comparative Nutrition (ESVCN 2019), Turin, Italy, 17-20 September 2019. https://doi.org/10.5281/ZENODO.3333395

JOHNSON, R., et al. Towards a competitive and sustainable OA market in Europe - A study of the open access market and policy environment. OpenAIRE, 2017. http://eprints.whiterose.ac.uk/114081/

JOHNSON, R., WATKINSON, A., MABE, M. The STM Report. An overview of scientific and scholarly publishing. STM International Association of Scientific, Technical and Medical Publishers, 2018. https://www.stm-assoc.org/2018 1004 STM Report 2018.pdf

LARIVIÈRE, V., HAUSTEIN, S., MONGEON, P., « The Oligopoly of Academic Publishers in the Digital Era ». PLOS ONE, 2015, vol. 10, nº 6, e0127502. https://doi.org/doi:10.1371/journal.pone.0127502

MABE, M. « L'édition des revues scientifiques ». In : J. Schöpfel (Ed.), La Publication Scientifique. ISTE, 2008. p. 51-68.

MCSHEA, J. Science, Technology and Healthcare: 2018 - Market Size, Share, Forecast and Trend Report. Outsell Inc, 2018. https://www.outsellinc.com/product/science-technologyand-healthcare-2018/

MEHRA, V., "Challenges facing non-English speaking scholars ». Research Information, 2019, $n^{\circ} 103$, p. 22-24. https://www.researchinformation.info/analysis-opinion/challenges-facing-non-englishspeaking-scholars

MIROWSKI, P., " The future(s) of open science ". Social Studies of Science, 2018, vol. 48, n ${ }^{\circ}$ 2, p. $171-$ 203. https://doi.org/10.1177/0306312718772086

OST. La position scientifique de la France dans le monde, 2000-2015. Observatoire des Sciences et Techniques, 2018. https://www.hceres.fr/fr/Rapport-PSF

OST. Dynamics of scientific production in the world, in Europe and in France, 2000-2016. Observatoire des Sciences et Techniques, 2019. https://www.hceres.fr/fr/publications/dynamics-scientificproduction-world-europe-and-france-2000-2016-ost

OUROUK. Des modèles économiques et de financement des services d'IST. Etude COPIST 5. CNRS, 2018. http://adbu.fr/les-etudes-du-copist-catalogue-doffres-partagees-en-ist/

PILLOT, J., MARTY, F., "La gratuité, casse-tête du régulateur face aux GAFAM ». The Conversation, 18 septembre 2019. https://theconversation.com/la-gratuite-casse-tete-du-regulateur-face-aux-gafam$\underline{123700}$

PIWOWAR, H. et al., "The state of OA: a large-scale analysis of the prevalence and impact of Open Access articles ». PeerJ, 2018, n 6, e4375. https://doi.org/10.7717/peeri.4375

POSADA, A., CHEN, G. Inequality in Knowledge Production: The Integration of Academic Infrastructure by Big Publishers. In: ELPUB 2018, Jun 2018, Toronto, Canada. Toronto. https://hal.archivesouvertes.fr/hal-01816707v1

RENTIER, B. Open science, the challenge of transparency. Académie royale de Belgique, 2019. https://academie-editions.be/accueil/369-open-science-the-challenge-of-transparency.html

ROBINSON-GARCIA, N., COSTAS, R., VAN LEEUWEN, T. N. Indicators of Open Access for universities. In : ISSI 2019, Rome, 2-5 September, 2019. https://arxiv.org/abs/1906.03840

SCHIMMER, R., GESCHUHN, K. K., VOGLER, A. Disrupting the subscription journals' business model for the necessary large-scale transformation to open access. A Max Planck Digital Library open access 
policy white paper. Max Planck Digital Library, 2015. http://hdl.handle.net/11858/00-001M-0000$\underline{0026-C 274-7}$

SCHONFELD, R. C., "Big Deal: Should Universities Outsource More Core Research Infrastructure? » Ithaka $S+R$, 4 January 2018. https://doi.org/10.18665/sr.306032.

SCHÖPFEL, J., PROST, H. The scope of open science monitoring and grey literature. In : 12th Conference on Grey Literature and Repositories, National Library of Technology (NTK), 17 October 2019, Prague, Czech Republic.

SCHÖPFEL, J., FARACE, D., PROST, H., ZANE, A. Data Papers as a New Form of Knowledge Organization in the Field of Research Data. In : Colloque ISKO France 2019, 9-11 Octobre 2019, Montpellier.

SCIENCE-METRIX. Analytical Support for Bibliometrics Indicators Open access availability of scientific publications. Science-Metrix Inc, 2018. http://www.science-metrix.com/sites/default/files/sciencemetrix/publications/science-metrix open access availability scientific publications report.pdf

SOTUDEH, H. GHASEMPOUR, Z., "The world's approach toward publishing in Springer and Elsevier's APC-funded open access journals ». College \& Research Libraries, 2018, vol. 79, n² 2, p. 257-278. https://doi.org/10.5860/crl.79.2.257

SUBER, P. Open access. MIT Press, 2012. http://mitpress.mit.edu/books/open-access

VAJOU, M., MARTINEZ, R., CHAUDIRON, S., "Les enjeux économiques de l'édition scientifique, technique et médicale : Analyses et questions clés ». Les Cahiers du Numérique, 2009, vol. 5, n² 2, p. 143-172. https://www.cairn.info/revue-les-cahiers-du-numerique-2009-2-page-143.htm\#

VANHOLSBEECK, M., "La notion de Science Ouverte dans l'Espace européen de la recherche ". Revue française des sciences de l'information et de la communication, 2017, $\mathrm{n}^{\circ} 11$. http://journals.openedition.org/rfsic/3241

WANG, Y., HU, R., LIU, M., " The geotemporal demographics of academic journals from 1950 to 2013 according to Ulrich's database ». Journal of Informetrics, 2017, vol. 11, $\mathrm{n}^{\circ} 3$, p. 655-671. https://doi.org/10.1016/j.joi.2017.05.006

WILKINSON, M., et al., " The FAIR Guiding Principles for scientific data management and stewardship ». Scientific Data, $\mathrm{n}^{\circ}$ 3, 160018. https://doi.org/doi:10.1038/sdata.2016.18

WILLIAMS, A., "Altmetrics: An Overview and Evaluation ». Online Information Review, 2016, vol. 41, n 3, p. 311-317. https://doi.org/doi:10.1108/OIR-10-2016-0294

WISE, A., ESTELLE, L. Society Publishers Accelerating Open access and Plan S (SPA-OPS) project. Information Power, 2019. https://doi.org/10.6084/m9.figshare.c.4561397.v2 